\title{
Piomiositis, sacroiliitis y espondilodiscitis por Staphylococcus hominis en una mujer sin compromiso inmune
}

\author{
N. GÓMEZ RODRÍGUEZ, O. DURÁN MUÑOZ1 \\ Servicios de Reumatología $y^{\prime}$ Medicina Interna. Hospital POVISA. Vigo (Pontevedra)
}

\section{RESUMEN}

Las infecciones osteoarticulares por estafilococos coagulasa negativos son raras en ausencia de factores de riesgo. Presentamos una paciente de 73 años, sin evidencia de compromiso inmune, que desarrolló piomiositis, sacroiliitis izquierda y espondilodiscitis involucrando las dos primeras vértebras dorsales por Staphylococcus hominis. El cuadro clínico infeccioso se desarrolló durante las cinco semanas que siguieron la administración de antiinflamatorios no esteroideos por vía intramuscular a causa de dolor lumbosacro asociado a una hernia discal L4-L5. Esta es la primera descripción conocida de una infección musculoesquelética multifocal por Staphylococcus hominis en una paciente no inmunocomprometida.

PALABRAS CLAVE: Staphylococcus hominis. Estafilococo coagulasa negativo. Espondilodiscitis infecciosa. Piomiositis. Sacroiliitis infecciosa.
PYOMYOSITIS, SACROILIITIS AND SPONDYLODISCITIS CAUSED BY STAPHYLOCOCCUS HOMINIS IN A IMMUNOCOMPETENT WOMAN

\begin{abstract}
In abscense of risk factors, osteoarticular infections by coagulasenegative staphylococci are very infrequent. We described the case of a immunocompetent 73-year-old-woman that suffered pyomyositis, left sacroiliitis and spondylodiscitis involving the first and second thoracic vertebrae by Staphylococcus hominis. This multifocal infection occurred five-weeks after intramuscular administration of NSAI for treatment of low back pain associated with a herniated disc L4-L5. This is the first know case of a multifocal muscleskeletal infection by Staphylococcus hominis in a patient immunocompetent.
\end{abstract}

KEY WORDS: Staphylococcus hominis. Coagulase-negative staphylococci. Pyomyositis. Infectious Spondylodiscitis. Septic sacroiliitis.

N. Gómez Rodríguez, O. Durán Muñoz. Piomiositis, sacroiliitis y espondilodiscitis por Staphylococcus hominis en una mujer sin compromiso inmune. An Med Interna (Madrid) 2006; 23: 582-584.

\section{INTRODUCCIÓN}

En las espondilodiscitis y en las artritis infecciosas, la principal vía de acceso de los microorganismos es la hematógena. La etiología está dominada por los estafilococos, responsables del 80-90\% de los casos. El género Staphylococcus consta de más de 32 especies y varias subespecies, la mayoría aerobias facultativas, entre las que destaca Staphylococcus aureus, que se diferencia del resto por producir la enzima coagulasa. En el pasado, los estafilococos coagulasa negativos rara vez provocaban infecciones de relevancia clínica, pero el empleo de catéteres y la colocación de prótesis ha incrementado su capacidad patógena, sobre todo en pacientes inmunodeprimidos. Las dos especies más aisladas son $S$. epidermidis y S. saprophyticus $(1,2)$. S. hominis es un estafilococo coagulasa negativo que rara vez produce infecciones en pacientes sin esos factores de riesgo. Por este motivo, hemos considerado oportuno describir el caso una paciente inmunocompetente que desarrolló piomiositis, sacroiliitis izquierda y espondilodiscitis por esta especie de estafilococo coagulasa negativo cuya puerta de entrada fue probablemente una inyección intramuscular. Adicionalmente, revisamos la literatura sobre espondilodiscitis y sacroiliitis por estafilococos coagulasanegativos mediante Medline-Embase-Plus a lo largo del periodo 1978-2005 utilizando como palabras clave: vertebral osteomyelitis, infectious spondylodiscitis / spondylodiskitis, coagulase-negative staphylococci, sacroiliitis y Staphylococcus hominis.

\section{CASO APORTADO}

Mujer de 73 años, sin antecedentes personales de interés, que ingresa a causa de dolor dorsal y en el área glútea izquierda, febrícu-

Trabajo aceptado: 8 de junio de 2006 
la vespertina, así como pérdida progresiva de fuerza en las extremidades inferiores. Los síntomas se habían iniciado cinco semanas antes, después de un episodio de dolor lumbar tratado con antiinflamatorios intramusculares y miorrelajantes.

En la exploración destacaba una temperatura axilar de $37,6^{\circ} \mathrm{C}$, una escoliosis dorsal sinistroconvexa, una marcha anormal por dolor en el área glútea izquierda, que estaba empastada a la palpación, dolor intenso a la presión y percusión sobre las dos primeras vértebras dorsales, restricción de los movimientos de rotación dorsal, dolor en el miembro inferior derecho con las maniobras de elongación radicular (elevación en extensión dolorosa a $45^{\circ}$, Lasègue y Bragard positivos), amiotrofia de interóseos y flexores de las manos e hiperreflexia en las cuatro extremidades.

Los análisis mostraron anemia normocrómica microcítica $(\mathrm{Hb}: 88$ g/L; VCM: $81 \mathrm{Fl})$, leucocitosis (19,5 x 10\%/L; 85\% neutrófilos), elevación de VSG $\left(121 \mathrm{~mm} / 1^{\mathrm{a}} \mathrm{h}\right.$; valores normales $\left.<20\right)$ y de la proteína $\mathrm{C}$ reactiva $(131 \mathrm{mg} / \mathrm{L}, \mathrm{VN}<5)$. Las concentraciones séricas de glucosa, urea, creatinina, amilasa, fosfatasa alcalina, transaminasas, creatíncinasa, calcio, fosforo, sodio, potasio, hierro y cloro fueron normales. La ferritina fue de $584 \mathrm{ng} / \mathrm{ml}$. En el proteínograma se objetivó hipoalbuminemia (2,5 g /L; VN: 3-5), elevación de la alfa-2-globulina y gammaglobulinas. El estudio de coagulación, la dosificación de inmunoglobulinas, las crioglobulinas, la serología tifo-paratífica y brucelar, los anticuerpos frente al VIH y la intradermorreacción de Mantoux (RT-23) fueron normales o negativos.

En la proyección de la radiografía de tórax se observaba desaparición del espacio D1-D2. Una tomografía computarizada lumbosacra demostró una listesis de grado I de L4 sobre L5, una protrusión discal paramedial derecha L4-L5, irregularidad de la interlínea sacroilíaca izquierda asociada a aumento de partes blandas en su porción anterior y un área hiperdensa entre el glúteo y el piramidal izquierdos, compatible con un absceso (Fig. 1). La resonancia dorsal puso de manifiesto hiperseñal en las secuencias ponderadas en T2 e hiposeñal en las ponderadas en T1, involucrando los cuerpos vertebrales, bastante desestructurados, de las dos primeras vértebras dorsales, así como una imagen sugestiva de absceso paravertebral (Fig. 2). Una gammagrafía ósea con Tc99m localizó dos focos de intensa hipercaptación, uno en D1-D2 y otro en la sacroilíaca izquierda.

Se procedió a un desbridamiento quirúrgico de la espondilodiscitis dorsal y a la fijación cervicodorsal (Hartshill de C6 a D4). En las muestras tisulares obtenidas (músculo y hueso necrótico) se visualizaron microorganismos grampositivos que fueron posteriormente recuperados e identificados como Staphylococcus hominis resistente

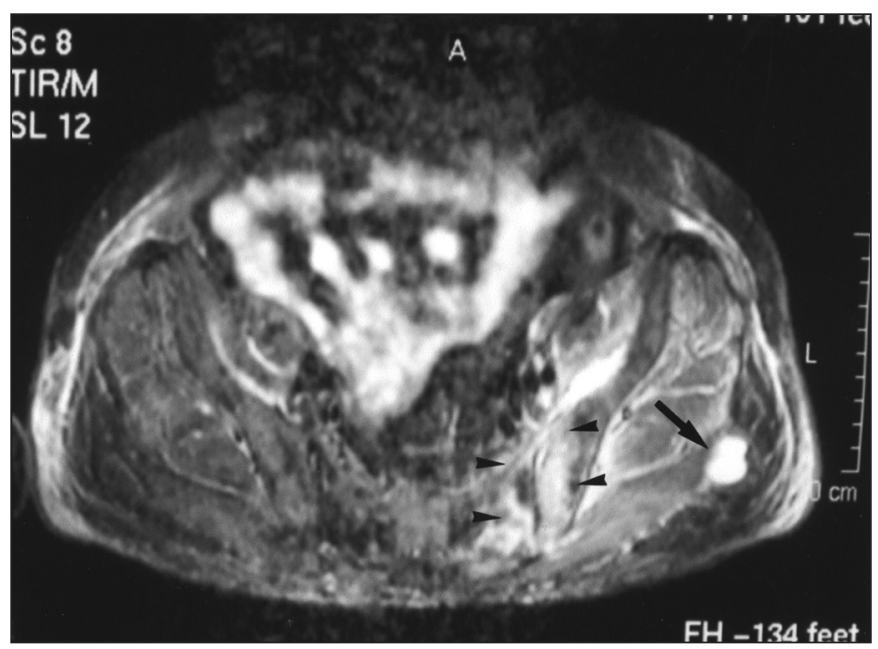

Fig. 1. Corte axial de la resonancia magnética de la paciente, ponderada en T2, a la altura del absceso glúteo izquierdo (flecha). Puede observarse un edema intenso (hiperseñal) de los músculos glúteos y de las dos vertientes óseas de la sacroilíaca ipsilateral (puntas de flecha), que reflejan una sacroiliitis.

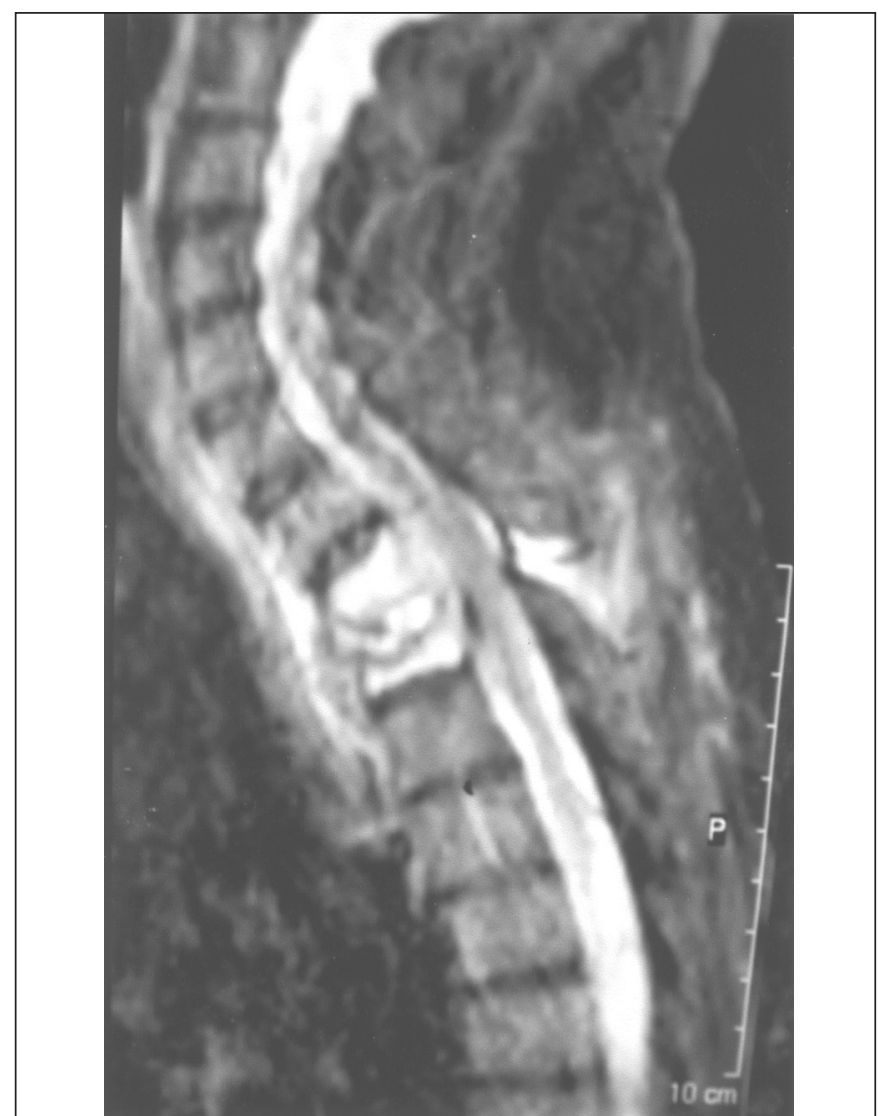

Fig. 2. Corte sagital de la resonancia magnética del raquis cervicodorsal ponderada en $T 2$ en el que se observa hiperseñal e irregularidad de la plataforma epifisaria de la vértebra D1 y del espacio discal D1-D2; la mitad superior de D2 ha sido destruida por el proceso infeccioso

a la oxacilina. La misma bacteria se obtuvo del absceso glúteo izquierdo, drenado por vía percutánea. El estudio ecocardiográfico no demostró signos de endocarditis.

Se administró tratamiento intravenoso con vancomicina (500 $\mathrm{mg} / 6$ horas) durante 5 semanas y rifampicina oral durante otras 3 semanas (300 mg/8 horas). La evolución fue favorable con mejoría clínica y radiológica de la espondilodiscitis, piomiositis y sacroiliitis izquierda. La enferma fue seguida durante tres años, quedando como secuelas una discreta paresia en los miotomas correspondientes a C7 y C8 bilateralmente y dolor cervicodorsal de intensidad leve y características mecánicas, que no interfiere con las actividades de la vida diaria.

\section{DISCUSIÓN}

Los estafilococos coagulasa-negativos causan el 3-18\% de las espondilodiscitis infecciosas (3-7), porcentaje que asciende hasta el $25-30 \%$ en las formas postquirúrgicas $(8,9)$ y cuando coexiste una endocarditis infecciosa (10). Pese a que sólo el 4-15\% de los pacientes con endocarditis presentan focos de osteomielitis vertebral, éstos suelen constituir el debut clínico (10). Por otra parte, únicamente el 2-3\% de las sacroiliitis piógenas son producidas por estafilococos coagulasa negativos (11).

La paciente descrita presentaba piomiositis, sacroiliitis izquierda y espondilodiscitis dorsal por Staphylococcus hominis. En la revisión de la literatura no hemos hallado ningún 
caso de infección musculoesquelética multifocal por estafilococos coagulasa negativos en pacientes inmunocompetentes. Tampoco hemos hallado descripciones de espondilodiscitis piógenas por $S$. hominis, aunque en algunos artículos no se especifica la subespecie de estafilococo coagulasa-negativo $(3,9,12)$. Esta revisión nos ha permitido identificar 73 casos de espondilodiscitis por estafilococos coagulasa negativos, siendo la subespecie S. epidermidis responsable del $72 \%$, seguida de lejos por S. lugdunensis con el 7\% (3-10,12-26). La mayoría de los enfermos presentaba factores de riesgo generales como diabetes, neoplasias, insuficiencia renal crónica en hemodiálisis, cirrosis hepática, conectivopatías o inmunodepresión. Dentro de las puertas de entrada destacaron los catéteres intravenosos y otros dispositivos intravasculares $(5,12,13,23)$, así como la inoculación yatrogénica en procedimientos quirúrgicos sobre el raquis $(3,8,9)$. La mayoría de las espondilodiscitis afectaron el segmento lumbar e involucraron sólo un segmento móvil, siendo excepcionales las formas multifocales (24).

Además de la etiología, Staphylococcus hominis, en nuestra paciente cabe destacar la ausencia de factores sistémicos predisponentes y el probable mecanismo patogénico de la infección musculoesquelética multifocal. Así, consideramos que las inyecciones intramusculares de antiinflamatorios no esteroideos constituyeron la puerta de entrada hasta el glúteo izquierdo, en cuyo seno se desarrolló una piomiositis. La sacroiliitis podría haberse producido por contigüidad, aunque también es factible la diseminación hematógena, mecanismo que explicaría la espondilodiscitis dorsal. No puede descartarse que la necrosis muscular por inyecciones intramusculares repetidas en la misma localización y un inóculo elevado de Staphylococcus hominis hayan creado las condiciones adecuadas para el desarrollo de este cuadro infeccioso.

\section{Bibliografía}

1. Kloss WE, Bannerman TL. Staphylococcus and Micrococcus. En: Murray PR, Baron EJ, Pfaller MA, Tenover FC, Yolken RH, eds. Manual of Clinical Microbiology. 7th Edition. Washington DC. ASM Press; 1999. p. 264-282

2. Archer GL. Staphylococcus epidermidis and other coagulase-negative staphylococci. En: Mandell G L, Bennet J E, Dolin R, eds. Mandell, Douglas and Bennett's principles and practice of infectious diseases, 5th Edition. Philadelphia: Churchill Livingstone; 2000. p. 2092-2100.

3. Belzunegui J, Del Val N, Intxausti JJ, De Dios JR, Queiro R, González C, et al. Vertebral osteomyelitis in northern Spain. Report of 62 cases. Clin Exp Rheumatol 1999; 17: 447-452.

4. Stefanovski N, Van Voris LP. Pyogenic vertebral osteomyelitis: report of a series of 23 patients. Contemp Orthop 1995; 31: 159-164.

5. Nolla JM, Ariza A, Gómez-Vaquero C, Fiter J, Bermejo J, Valverde J, et al. Spontaneous pyogenic vertebral osteomyelitis in nondrug users. Sem Arthritis Rheum 2002; 31: 271-278.

6. Patzakis M, Rao S, Wilkins J, Moore TM, Harvey P. Analysis of 61 cases of vertebral osteomyelitis. Clin Orthop 1991; 264: 178-183.

7. Carragee EJ. Pyogenic vertebral osteomyelitis. J Bone Joint Surg 1997; 79A; 874-880.

8. Jiménez-Mejías ME, de Dios Colmenero J, Sánchez-Lora FJ, Palomino Nicas J, Reguera JM, García de la Heras J, et al. Postoperative spondylodiskitis: etiology, clinical findings, prognosis, and comparison with nonoperative pyogenic spondylodiskitis. Clin Infect Dis 1999; 29: 339345.

9. Dufour V, Feydy A, Rillardon L, Redondo A, Le Page L, Bert F, et al. Comparative study of postoperative and spontaneous pyogenic spondylodiscitis. Semin Arthritis Rheum 2005; 34: 766-771.

10. Le Moal G, Roblot F, Paccalin M, Sosner P, Burucoa C, Roblot P, et al. Clinical and laboratory characteristics of infective endocarditis when associated with spondylodiscitis. Eur J Clin Infect Dis 2002; 21: 671-675.

11. Zimmermann III B, Mikolich DJ, Lally EV. Septic sacroiliitis. Sem Arthritis Rheum 1996; 26: 592-604.

12. Bucher E, Trampuz A, Donati L, Zimmerli W. Spondylodiscitis associated with bacteraemia due to coagulase-negative Staphylococci. Eur J Clin Microbiol Infect Dis 2000; 19: 118-120.

13. Parker MA, Tuazon CU. Cervical osteomyelitis. Infection due to Staphylococcus epidermidis in hemodialysis patients. JAMA 1978; 240: 50-51

14. Tuazon CU, Miller H. Clinical and microbiologic aspects of serious infections caused by Staphylococcus epidermidis. Scand J Infect Dis 1983; 15: 347-360.

15. Karthigasu KT, Bowman RA, Grove DI. Vertebral osteomyelitis due to Staphylococcus warneri. Ann Rheum Dis 1986; 45: 1029-1030.

16. Bryan CS, Parisi JT, Strike DG. Vertebral osteomyelitis due to Staphylococcus warneri attributed to a Hickman catheter. Diagn Microbiol Infect Dis 1987; 8: 57-59.

17. Mundlein E, von Braum H, Geiss HK, Springsklee M, Zeier M, Adrassy $\mathrm{K}$. Life-theatening infection wtih multiresistant Staphylococcus epidermidis in a patient with end-stage renal disease: cure with chloramphenicol and quinupristin/dalfopristin (RP 59500). Infection 1997; 25: 252254.

18. De Wit D, Mulla R, Cowie MR, Mason JC, Davies KA. Vertebral osteomyelitis due to Staphylococcus epidermidis. Br J Rheumatol 1993; 32 339-341.

19. Razonable RR, Lewallen DG, Patel R, Osmon DR. Vertebral osteomyelitis and prosthetic joint infection due to Staphylococcus simulans. Mayo Clin Proc 2001; 76: 1067-1070.

20. Greig JM, Wood MJ. Staphylococcus lugdunensis vertebral osteomyelitis. Clin Microbiol Infect 2003; 9: 1139-1141.

21. Gosbell I, Gottlieb T, Bradbury R. Native valve endocarditis and vertebral osteomyelitis caused by Staphylococcus epidermidis. Med J Aust 1992; 156: 662

22. Wood CA, Sewell DL, Strausbaugh LJ. Vertebral osteomyelitis and native valve endocarditis caused by Staphylococcus warneri. Diagn Microbiol Infect Dis 1989; 12: 261-263.

23. Valero R, Castañeda O, de Francisco ALM, Piñera C, Rodrigo E, Arias M. Sospecha clínica de osteomielitis vertebral: dolor de espalda en los pacientes con infección asociada a catéter de hemodiálisis. Nefrología 2004; $24: 583-588$

24. Announ N, Mattei JP, Jaoua S, Fenollar F, Sati H, Chagnaud C, et al Multifocal discitis caused by Staphylococcus warneri. Rev Rhum 2004; 71: 240-242.

25. Murdoch DR, Everts RJ, Chambers ST, Cowan IA. Vertebral osteomyelitis due to Staphylococcus lugdunensis. J Clin Microbiol 1996; 34 993-994.

26. Kragsbjerg P, Bomfim-Loogna J, Tornqvist E, Soderquist B. Development of antimicrobial resistance in Stapylococcus lugdunensis during treatment: report of a case of bacterial arthritis, vertebral osteomyelitis and infective endocarditis. Clin Microbiol Infect 2000; 6: 496-499. 